\title{
Una revisión sistemática de la literatura enfocada al uso de vehículos aéreos no tripulados durante el proceso de detección de vegetación
}

\author{
Enrique Ponce-Corona ${ }^{1}$, María Guadalupe Sánchez ${ }^{1}$, Daniel Fajardo-Delgado ${ }^{1}$, \\ Brenda Acevedo-Juárez ${ }^{2}$, Miguel De-la-Torre ${ }^{3}$, Himer Avila-George ${ }^{3}$, Wilson Castro ${ }^{4}$ \\ eponceo8@gmail.com,msanchez@itcg.edu.mx, dfajardo@itcg.edu.mx, \\ brenda.acevedo@academicos.udg.mx,miguel.dgomora@academicos.udg.mx, \\ himer.avila@academicos.udg.mx, wcastro@unf.edu.pe
}

${ }^{1}$ Departamento de Sistemas y Computación, TecNM - Instituto Tecnológico de Ciudad Guzmán, Ciudad Guzmán 49100, Jalisco, México.

${ }^{2}$ Departamento de Ciencias Naturales y Exactas, Universidad de Guadalajara, Ameca 4660o, Jalisco, México.

${ }^{3}$ Departamento de Ciencias Computacionales e Ingenierías, Universidad de Guadalajara, Ameca 4660o, Jalisco, México.

${ }^{4}$ Facultad de Ingeniería de Industrias Alimentarias, Universidad Nacional de Frontera, Sullana 20100, Perú.

DOI: 10.17013/risti.36.82-101

Resumen: En los últimos años, se ha popularizado el uso de vehículos aéreos no tripulados en el desarrollo de aplicaciones agrícolas y forestales. Debido a la importancia de los cultivos tanto en entornos rurales como urbanos, la extracción de información a partir de datos obtenidos por sensores remotos es un área de investigación relevante. Dicha información es fundamental para realizar la planeación urbana y rural, establecer de nichos ecológicos sustentables, estimar el rendimiento de cultivos, realizar inventarios forestales, supervisar los sistemas de gestión agrícola y algunas otras aplicaciones agrícolas. En este artículo, se presenta una revisión sistemática de la literatura referente al uso de los vehículos aéreos no tripulados en aplicaciones relacionadas con la detección de vegetación y el inventario de plantas. Asimismo, se realiza un análisis de las principales técnicas computacionales y estadísticas utilizadas para el procesamiento de las imágenes tomadas desde vehículos aéreos no tripulados.

Palabras-clave: Vehículos aéreos no tripulados; vegetación; conteo; inventario; procesamiento de imágenes.

\section{A systematic review of the literature focused on the use of unmanned aerial vehicles during the vegetation detection process}

Abstract: In recent years, the use of unmanned aerial vehicles has become popular in the development of agricultural and forestry applications. Due to the importance of plants in both rural and urban environments, the extraction of information from 
remote sensing data is a relevant research area; such information is fundamental for urban and rural planning, establishing sustainable ecological niches, estimating crop yields, conducting forest inventories, monitoring agricultural management systems and some other agricultural applications. In this article, a systematic review of the literature concerning the use of unmanned aerial vehicles in applications related to vegetation detection and plant inventory is presented. Likewise, an analysis of the main computational and statistical techniques used for processing unmanned aerial vehicles images is also made.

Keywords: Unmanned aerial vehicles; vegetation; counting; inventory; image processing; algorithms.

\section{Introducción}

Actualmente, la agricultura en todo el mundo tiene como objetivo garantizar la seguridad alimentaria presente y futura. Esto, motiva a investigadores, tecnólogos y agricultores a buscar y desarrollar nueva tecnología que ayude a optimizar los recursos en la producción de alimentos.

De acuerdo con González et al. (2016), la agricultura de precisión se basa en el manejo específico de una zona de cultivo. Para ello, se utilizan herramientas tecnológicas como el posicionamiento global (GPS), dispositivos de distribución de riego, fertilizantes y plaguicidas variables, redes de sensores $\mathrm{y}$, recientemente, vehículos aéreos no tripulados (VANT). Estas herramientas en conjunto realizan el proceso de recolección de la información, la cual es finalmente plasmada en mapas digitales sobre los cuales se toman decisiones sobre el manejo agronómicos de los cultivos. El objetivo principal de la agricultura de precisión es la obtención de mayores rendimientos económicos, sociales y medioambientales. Dicho enfoque se ha extendido en los últimos años como un método eficaz para obtener mayores rendimientos en la producción agrícola y optimizar el uso de recursos en dicho proceso.

Los VANT son aeronaves que vuelan autónomamente hasta una altura de $120 \mathrm{~m}$ usando un sistema de piloto automático. Los vuelos son monitoreados desde un centro de control en tierra. Según Escalante et al. (2016), fue el ejército estadounidense, en la década de los cincuenta, el primero en utilizar los VANT en aplicaciones militares relacionadas con tareas de reconocimiento, vigilancia, y mapeo.

En los últimos años, se ha popularizado el uso de VANT para el desarrollo de aplicaciones civiles en el área de percepción remota. Lo anterior se ha debido a los recientes avances tecnológicos en el desarrollo de sistemas de control de vuelo y navegación, así como en las mejoras en las propiedades de las cámaras. En este contexto, las imágenes tomadas desde un VANT se han incluido en varios estudios de percepción remota, principalmente en aplicaciones agrícolas y forestales (Koc-San et al., 2018).

Entre las principales aplicaciones en la agricultura se encuentran la detección, identificación, conteo de cultivos, e inventarios de árboles, bosques, hierbas y coníferas. El conteo de árboles, plantas o cultivos es una actividad importante para los agricultores en la toma de decisiones. De acuerdo con She et al. (2014), dicha actividad por lo regular se realiza de manera manual consumiendo tiempo y recursos, lo que obliga a los productores a sobreestimar los recuentos de plantas, árboles o cultivos, a realizarlos con 
poca frecuencia y tener censos inexactos; además, de no tener acceso a un inventario en tiempo real. Esta problemática puede ser atendida a través del análisis de imágenes de alta resolución. Sin embargo, las imágenes de alta resolución temporal son difíciles y costosas de obtener, ya sea por imágenes satelitales o usando aeronaves (Grenzdörffer et al., 2008).

La aparición de sistemas VANT cambió en gran medida el modo de trabajo de los sistemas convencionales y proporcionó a los productores de cultivos una plataforma área estable y de bajo costo para la percepción remota (She et al., 2014). Los sensores con los que cuenta los VANT pueden proporcionar imágenes de áreas que resuelven las principales deficiencias que actualmente tienen los sistemas de adquisición de imágenes (Hunt et al. 2010). Los VANT pueden usarse para observar las estructuras pequeñas, específicas y detalladas de los cultivos, a diferencia de otros sistemas; al respecto, Hadas et al,. (2019), mencionaron que la teledetección realizada con VANT permite realizar inventarios frecuentes de zonas agrícolas y obtener productos de gran calidad y alta resolución.

Este artículo tiene como objetivo establecer el estado actual sobre la detección de vegetación usando imágenes tomadas desde un VANT. Después de esta sección introductoria, el artículo se estructura de la siguiente manera: la sección 2 presenta una breve contextualización sobre los VANT y las aplicaciones agrícolas y forestales, la sección 3 describe el método de revisión sistemática realizado, la sección 4 presenta el análisis de los resultados, y finalmente, en la sección 5 se presentan las conclusiones y trabajos futuros.

\section{Contextualización}

Debido a la gran cantidad de plataformas aéreas consideradas como VANT, existen diversas maneras de clasificarlas, una de las más comunes es según el tipo de plataforma, es decir, ala fija o multirotor. Para saber las ventajas que ofrece cada plataforma, recomendamos al lector revisar (Tahar \& Ahmad, 2013).

De acuerdo con Wong (2001), el uso de VANT en actividades agrícolas data desde principio de la década de los 90, sin embargo, su popularización se ha dado en años recientes en aplicaciones relacionadas con el monitoreo de cultivos o áreas forestales.

Las imágenes adquiridas mediante fotogrametría VANT son una solución atractiva debido a su bajo costo de monitoreo ambiental y alta resolución espaciotemporal. Dichas imágenes generalmente son evaluadas y procesadas usando algún software específico (ej. Pix4DMapper, PhotoScan/Metashape, EnsoMOSAIC) el cual permite la generación de productos cartográficos tales como los ortomosaicos (ortofotos).

Un mosaico corresponde a un conjunto de imágenes que presentan áreas de traslape entre sí, las cuales son combinadas en una sola imagen para ampliar el rango de visión de la escena. Cuando el mosaico es corregido de las distorsiones causadas por el relieve del terreno y los objetos en el, se denomina ortomosaico.

Para extraer información desde un ortomosaico se utilizan desde técnicas relacionadas con la segmentación de las imágenes (ej. binarización, filtros, operadores morfológicos) 
hasta técnicas de aprendizaje máquina (ej. redes neuronales artificiales, máquinas de soporte vectorial, árboles de decisión, entre otras).

\section{Revisión sistemática de la literatura}

En esta sección se ilustra el método que se siguió para realizar la presente revisión sistemática de la literatura. El método utilizado se basa en las recomendaciones hechas en Kitchenham \& Charters (2007) y aplicado recientemente por Miramontes et al. (2016), Hernández et al. (2017), Muñoz et al. (2019), entre otros; dicho método consta de tres fases principales: planificación de la revisión, desarrollo de la revisión y reporte de resultados.

\subsection{Planificación de la revisión}

La planificación es la primera etapa de la revisión sistemática, en esta etapa se desarrolla el protocolo que guiará la revisión. En este trabajo se consideraron las siguientes actividades: 1) identificación de la necesidad de la revisión, 2) formulación las preguntas de investigación, 3) definición de la cadena de búsqueda y 4) selección de las fuentes de datos. Las cuales se describen brevemente a continuación.

\section{Identificación de la necesidad para realizar la revisión sistemática}

Con el abaratamiento de los VANT y los avances tecnológicos en sistemas de control de vuelo y navegación así como en el diseño de sensores más potentes, se ha popularizado el desarrollo de aplicaciones civiles basadas en el procesamiento de imágenes adquiridas desde VANT, principalmente en áreas relacionadas con el monitoreo de cultivos o áreas forestales. En este tipo de aplicaciones, la extracción de información a partir de las imágenes juega un rol fundamental, por lo tanto, en este trabajo se busca revisar qué técnicas de procesamiento de imágenes y extracción de objetos se han utilizado en las áreas de detección de vegetación, conteo de plantas e inventario de árboles.

\section{Establecimiento de las preguntas de investigación}

Se establecieron las siguientes preguntas de investigación.

- PIo1: ¿Cómo ha evolucionado el interés por usar VANT en la detección, identificación y conteo de plantas?

- PIo2: ¿Cuáles son las principales fuentes de información en el tema de conteo de plantas usando VANT?

- PIo3: ¿Cuáles son los principales problemas abordados con la detección de la vegetación usando imágenes tomadas desde VANT?

- PIo4: ¿Qué plantas han sido las más estudiadas usando imágenes adquiridas desde VANT?

- PIo5: ¿Qué técnicas son las más utilizadas para el conteo de plantas usando imágenes tomadas desde VANT?

- PIo6: ¿Qué tipos de imágenes se usan en estudios relacionados con la detección de plantas usando imágenes VANT? 


\section{Creación de la cadena de búsqueda}

Para la creación de la cadena de búsqueda, se identificaron las palabras clave; las cuales fueron extraídas de las preguntas de investigación. Se decidió dividir el concepto plantas en: bosques, árboles, cultivos, y plantas. Los principales problemas identificados fueron: detección, identificación, conteo e inventario de plantas/árboles. Como resultado: Las palabras clave seleccionados fueron las siguientes: Forest, Tree, Plant, Crop, Inventory, Detection, Counting, Identification, and "Unmanned Aerial Vehicle".

La cadena de búsqueda se obtuvo a partir del agrupamiento de las palabras clave y el uso de los operadores lógicos AND y OR; como resultado, se obtuvo la siguiente cadena:

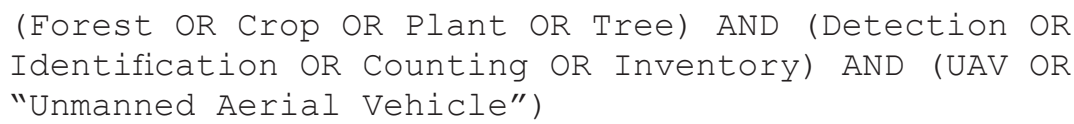

Para el presente estudio, se entiende por detección la acción de descubrir un tipo de cultivo, árbol o planta que no era patente en una imagen; identificación es el establecimiento de la clasificación taxonómica de una planta; conteo es la acción de contar objetos en una imagen, dichos objetos están asociados a arboles, plantas, surcos, e incluso frutas; finalmente, inventario consiste en la elaboración de un catálogo que recoge la diversidad de especies de plantas existentes en un área determinada.

\section{Identificación de las fuentes de datos}

Atendiendo las recomendaciones propuestas por Kitchenham \& Chartes (2007), sobre sus lecciones aprendidas para los procedimientos de búsqueda de datos; para este estudio, se decidió utilizar las siguientes fuentes de datos: (1) ACM Digital Library, (2) IEEE Xplore Digital Library, (3) ScienceDirect, Wiley y Scopus. Además, se decidió consultar MDPI dado su amplio contenido de acceso abierto en áreas relacionadas directamente con el presente estudio (percepción remota, sensores, y medioambiente).

\subsection{Desarrollo de la revisión}

El objetivo de esta segunda fase del método de revisión sistemática es identificar tantos estudios primarios como sea posible usando una estrategia de búsqueda imparcial; dichos estudios primarios permitirán responder a las preguntas de investigación. Para llevar a cabo el desarrollo de la presente revisión sistemática se deben realizar las siguientes dos actividades: (1) seleccionar los estudios primarios y (2) extraer y sintetizar la información.

\section{Seleccionar los estudios primarios}

Para garantizar que la estrategia de búsqueda de los estudios primarios sea imparcial, se definieron algunos criterios de inclusión y exclusión, y se siguió un procedimiento bien definido.

Criterios de inclusión. (CIo1) estudios en idioma inglés; (CIO2) estudios publicados durante el periodo comprendido entre enero del 2010 y diciembre de 2019; (CIo3) estudios que contengan al menos dos palabras clave en el título; (CIo4) estudios relacionados con la detección de plantas (inventario, conteo, identificación y detección). 
Criterios de exclusión. (CEO1) estudios duplicados; (CEO2) estudios que no se basen en el uso de imágenes tomadas desde vehículos aéreos no tripulados.

Procedimiento. Para seleccionar los estudios primarios se realizaron los siguientes pasos:

1. Adaptar la cadena de búsqueda al motor de la fuente de datos seleccionada.

2. Aplicar los criterios de inclusión CIo1 y CIO2.

3. Aplicar el criterio de inclusión CIo3.

4. Leer resumen, introducción y conclusiones, y aplicar el criterio de inclusión CI04 y los criterios de exclusión.

Según Wohlin (2014), el proceso de revisión sistemática puede ser ampliado usando un enfoque de búsqueda conocido como snowballing. El snowballing se refiere al uso de la lista de referencias de un documento para identificar documentos adicionales.

La Tabla 1, resume el número de trabajos de investigación publicados durante el periodo definido para el presente estudio (véase la columna 2), así como la selección de los estudios primarios (véase la columna 3). Durante el proceso de búsqueda, indexación, y clasificación varios estudios aparecieron duplicados. Para gestionar las referencias y eliminar los duplicados se utilizó la herramienta Mendeley. Tras aplicar el procedimiento definido para la selección de los estudios primarios se seleccionaron 88 estudios.

\begin{tabular}{lll} 
Fuentes de datos & $\begin{array}{l}\text { Resultados de la cadena de } \\
\text { búsqueda }\end{array}$ & Estudios primarios \\
\hline ScienceDirect & 91 & 25 \\
\hline IEEEXplore Digital Library & 47 & 21 \\
\hline ACM Digital Library & 2 & 1 \\
\hline Wiley & 16 & 1 \\
\hline Scopus & 79 & 20 \\
\hline MDPI & 85 & 20 \\
\hline Total & $\mathbf{3 2 0}$ & $\mathbf{8 8}$ \\
\hline
\end{tabular}

Tabla 1 - Número de investigaciones publicadas y estudios primarios

Finalmente, se llevó a cabo una etapa de snowballing y se verificaron las referencias de los estudios seleccionados para no perder ningún documento relevante; como resultado, se agregaron 2 artículos más. Por lo tanto, se seleccionaron en total 90 artículos para responder a las preguntas de investigación. El Anexo A muestra el listado de los estudios primarios.

\section{Extracción y síntesis de la información}

Antes de realizar la extracción de la información, los estudios primarios fueron organizados por medio de la herramienta de gestión de archivos PDF Mendeley. Para llevar a cabo la presente actividad se diseñó una hoja de cálculo donde se recopilaron 
los siguientes datos de cada estudio primario: título, autor, año, objetivo, tipo de vegetación, técnicas utilizadas, tipo de imagen, tipo de manuscrito, título de la fuente. Para extraer y analizar la información de todos los artículos, se siguieron los siguientes pasos: (1) La información fue extraída por el primer autor, y (2) por separado se verificó la información por los otros autores.

\section{Análisis de resultados}

En esta sección se muestran los resultados obtenidos de la revisión sistemática de la literatura, los cuales permiten conocer el panorama general de la detección de plantas usando imágenes adquiridas desde VANT. Los resultados se organizan por pregunta de investigación.

PIo1: ¿Cómo ha evolucionado el interés por usar VANT en la detección, identificación y conteo de plantas?

Los estudios identificados se analizaron durante la última década para conocer la frecuencia y la evolución del número de publicaciones. La Fig. 1 muestra los resultados de este proceso de análisis, allí se puede observar que recientemente se han incrementado notablemente la cantidad de estudios relacionados con la detección de vegetación utilizando imágenes VANT.

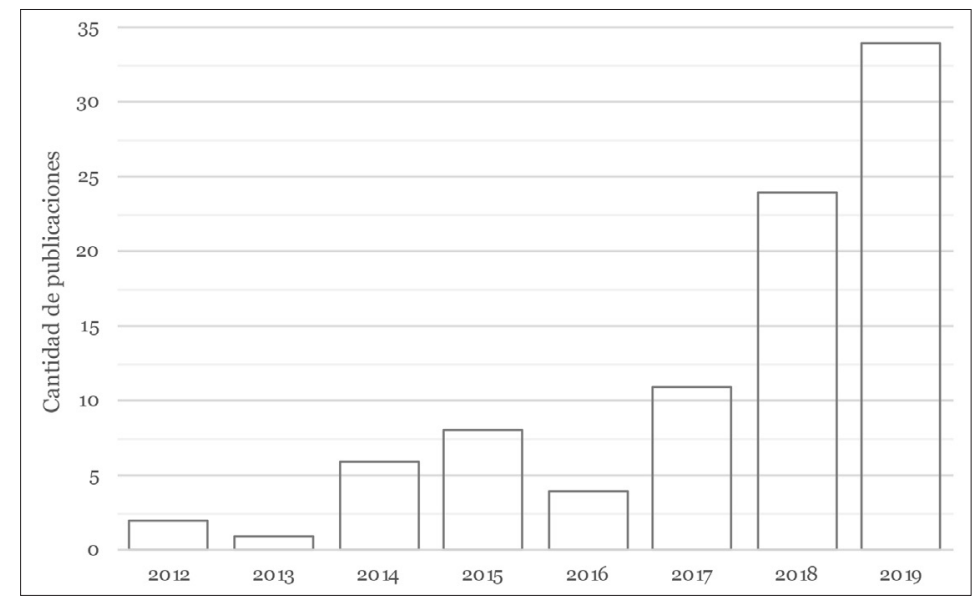

Figura 1 - Publicaciones por año

En lo que respecta a que países se encuentran desarrollando proyectos de investigación relacionadas con el uso de VANT como soporte a las actividades agrícolas y forestales destacan Brasil, China y España, ver la Fig. 2. En Brasil destacan sus estudios de cítricos y árboles frutales, en China sus estudios sobre coníferas, y en España el estudio de plantas de olivo. 


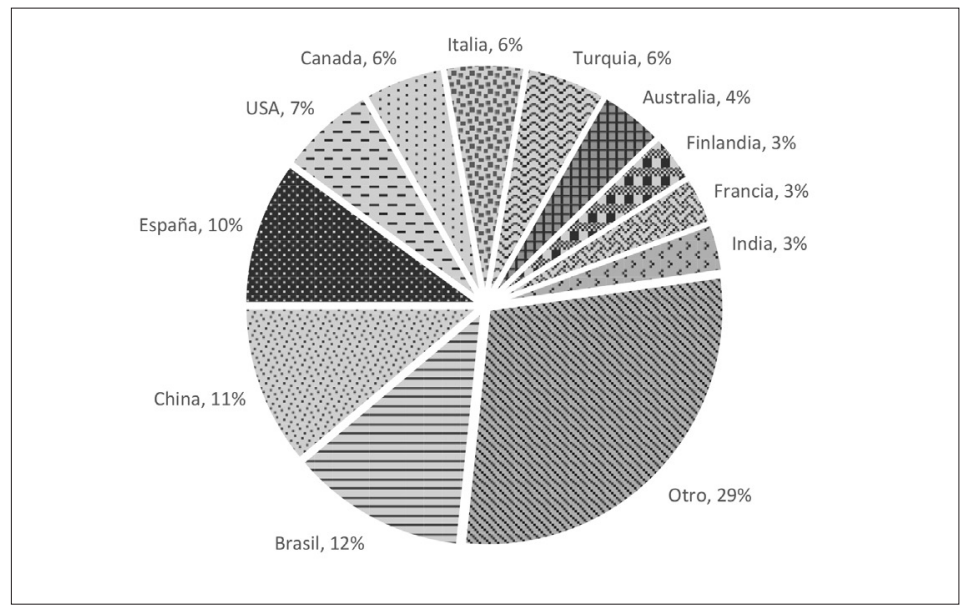

Figura 2 - Distribución de estudios primarios por país

PIo2: ¿Cuáles son las principales fuentes de información en el tema de conteo de plantas usando VANT?

De los 90 estudios que componen el conjunto de estudios primarios de la presente revisión sistemática, 70 fueron publicados en revistas y 20 en conferencias. En la Fig. 3, se muestran las revistas más activas en el área de detección de plantas usando imágenes VANT. Destaca las revistas Remote Sensing de la editorial MDPI y Computer and Electronics in Agriculture de la editorial Elsevier, en dichas revistas se han publicado 17 y 11 de los estudios seleccionados en esta revisión respectivamente.

PIo3: ¿Cuáles son los principales problemas abordados con la detección de la vegetación usando imágenes tomadas desde VANT?

La Fig. 4, muestra los tipos de problemas abordados mediante el procesamiento de imágenes adquiridas desde un VANT, los estudios se encuentran agrupados por el tipo de problema que solucionan (detección, identificación y conteo). Son 8 los estudios relacionados con problemas de conteo; 62 estudios se enfocan en la detección de plantas a nivel de bosques, plantaciones, surcos, árboles, arbustos, e incluso frutos; finalmente, 20 estudios se centran en la identificación de plantaciones, hierba y árboles.

PIo4: ¿Qué plantas han sido las más estudiadas usando imágenes adquiridas desde VANT?

En la Fig. 5, se muestra un gráfico de pastel el cual proporciona la información referente al tipo de planta que se han estudiado, se observa que la detección de maleza es lo que se ha estudiado más usando imágenes VANT; los árboles de cítricos, palmeras y la vid también han sido ampliamente estudiados. 


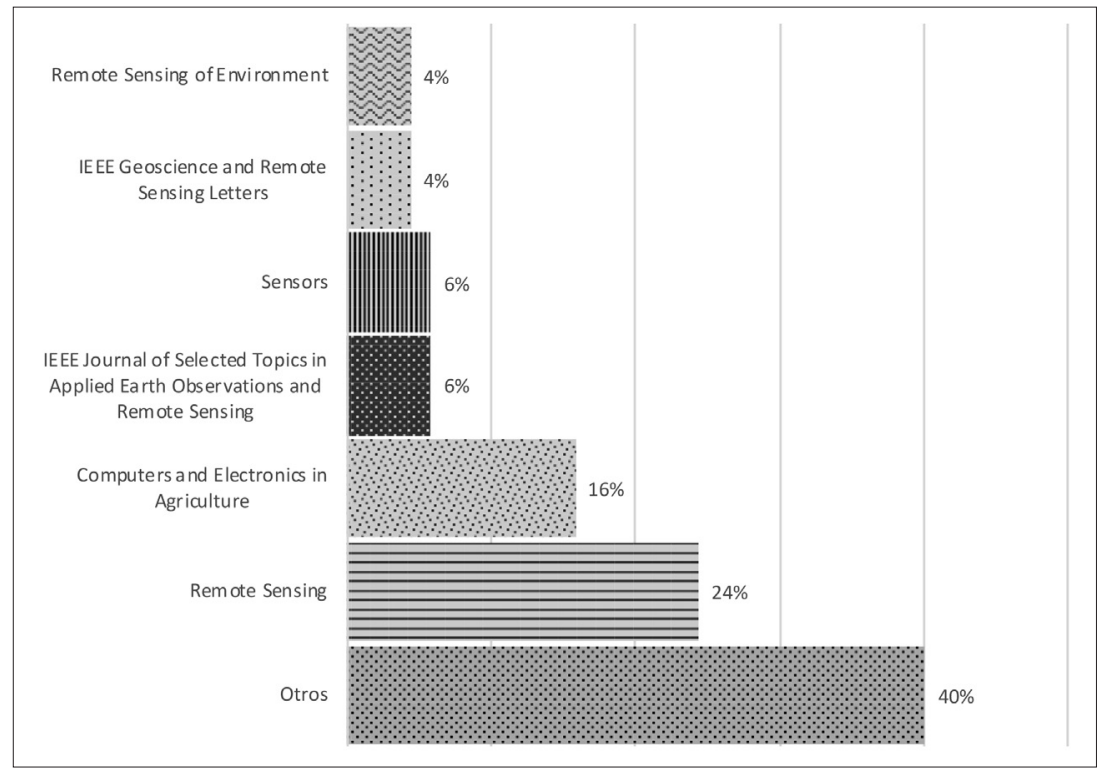

Figura 3 - Revistas en las cuales se ha publicado alguno de los estudios primarios

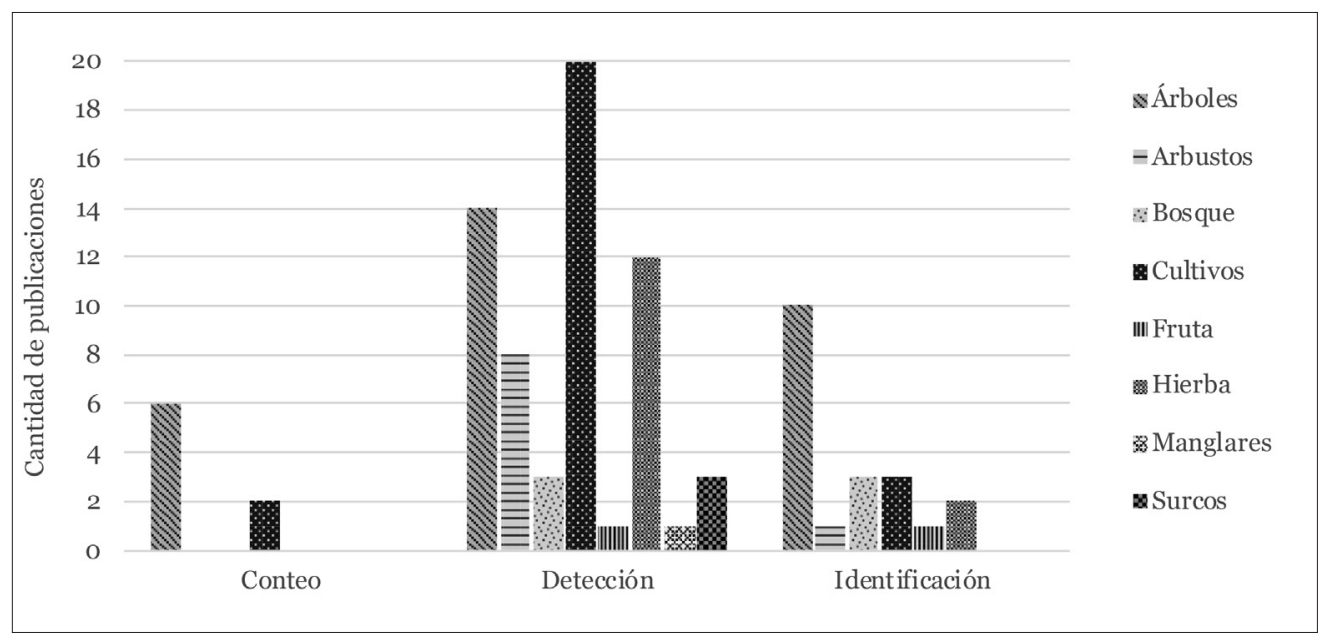

Figura 4 - Tipos de problemas abordados

PIo5: ¿Qué técnicas son las más utilizadas para el conteo de plantas usando imágenes tomadas desde VANT?

Para atender el problema de conteo de plantas, se observó que la técnica más usada son las redes neuronales convolucionales (CNN, por sus siglas en inglés), en total 18 
estudios utilizaron dicha técnica. Es de resaltar que el primer estudio que se encontró fue publicado en 2017, ver EPo8. Otra técnica muy utilizada, son las máquinas de soporte vectorial (SVM, por sus siglas en inglés), dicha técnica fue utilizada en 7 estudios primarios. Las SVM, son una técnica madura de aprendizaje máquina y ampliamente utilizada en problemas de clasificación y regresión, por lo cual no es extraño que el primer estudio primario date del año 2012, véase el EP43; en dicho estudio se propone una SVM para detectar coronas de árboles.

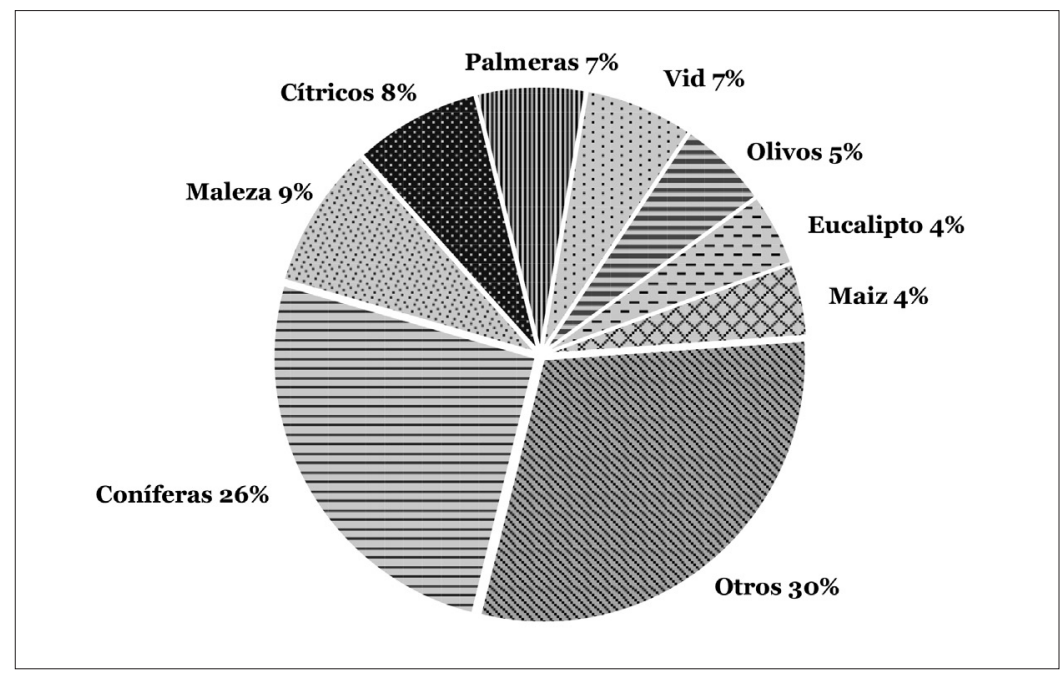

Figura 5 - Plantas estudiadas

Cabe mencionar que el software utilizado para procesar las imágenes es muy variado, desde lenguajes de programación de propósito general Matlab, Python y $\mathrm{C}++$, hasta software especializado como TerraScan, Agisoft PhotoScan y Pix4D.

PIo6: ¿Qué tipos de imágenes se usan en estudios relacionados con la detección de plantas usando imágenes VANT?

Los espacios de color juegan un papel fundamental en los estudios relacionados con el procesamiento de datos obtenidos desde sensores montados en VANT (De-la-Torre, 2019); entre los principales espacios de color se encuentran: RGB (por sus siglas en inglés Red, Green, Blue), HSV (por sus siglas en inglés Hue, Saturation, Value), y CIE Lab (también conocido como CIE $\mathrm{L}^{*} \mathrm{a}^{*} \mathrm{~b}^{*}$ ) el cual fue definido por la Comisión Internacional de Iluminación (CIE). En la Fig. 6, se muestra que el espacio de color más utilizado en los estudios primarios es el RGB, de hecho, más del 50\% de las investigaciones lo han utilizado; sin embargo, se observan algunos trabajos que utilizan los espacios de color tribanda CIE Lab y HSV. Por otra parte, se encontró que un 14\% de los estudios primarios utilizó imágenes multiespectrales y el 10\% datos LiDAR. Cabe mencionar que los estudios que basados en imágenes multiespectrales utilizaron principalmente las cámaras Canon S110 NIR, Tetracam mini-MCA-6 y Parrot Sequoia. 
Las imágenes hiperespectrales, a pesar de tener la ventaja de la gran cantidad de datos que contienen se han utilizado muy poco (2\%), tal vez debido al alto coste de los sensores de este tipo.

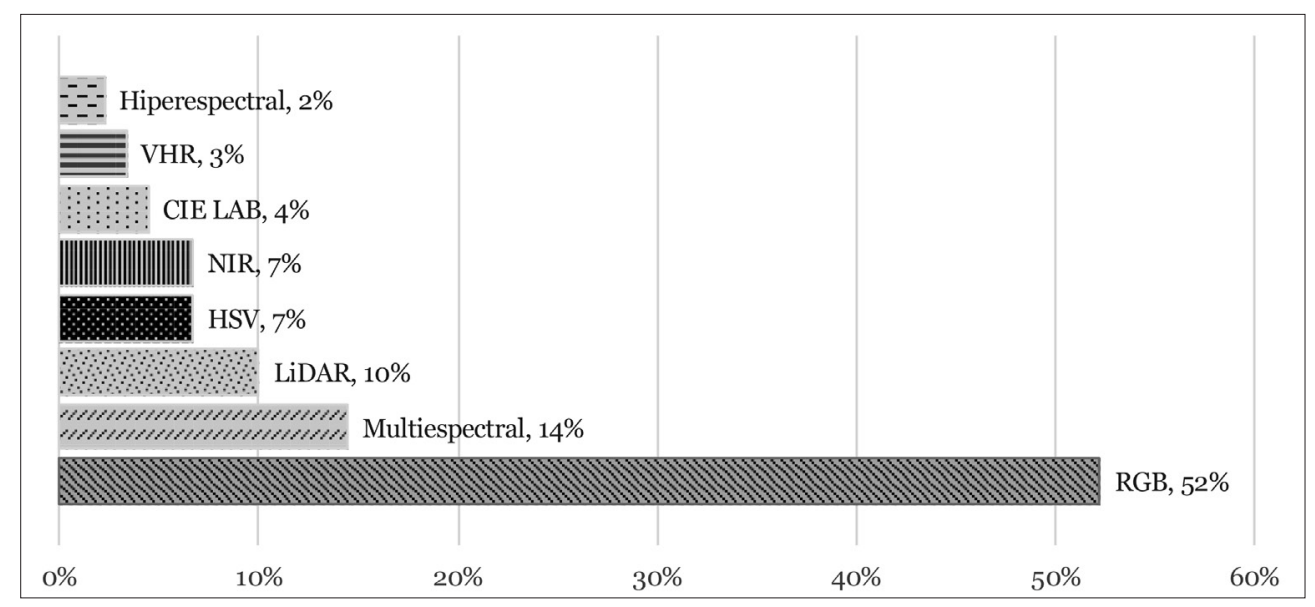

Figura 6 - Tipos de imágenes/datos utilizados

\section{Conclusiones}

La agricultura de precisión ha sido sustancialmente favorecida por las aplicaciones de la percepción remota en las últimas tres décadas. Sin embargo, la mayoría de los estudios se centran en la vigilancia de los cultivos. Se han hecho muchos esfuerzos para calcular parámetros como los índices de vegetación, rendimiento de los cultivos, la biomasa de las plantas, el estrés hídrico, entre otras.

En este trabajo de investigación, se realizó una revisión sistemática de la literatura referente al uso de VANT en aplicaciones agrícolas y forestales, en especifico se abordó el tema de la detección/identificación/conteo de plantas usando imágenes adquiridas desde VANT.

Se observó, que desde el año 2012 dada la popularidad que han alcanzado los VANT y los avances tecnológicos en el desarrollo de sensores, se han venido desarrollando cada vez más aplicaciones relacionadas con la detección de plantas. Los estudios abarcan diferentes niveles de granularidad, desde la detección de bosques, parcelas, surcos, hasta la identificación de árboles, arbustos, hierba, (incluso frutas y espigas) pasando por el conteo de plantas y la realización de inventarios de árboles.

Brasil, China, España y Estados Unidos de América son los países donde se han desarrollado la mayoría de las investigaciones reportadas en la presente revisión sistemática. La mayoría de los estudios primarios (78\%) fueron publicados en revistas científicas, siendo las revistas Remote Sensing de la editorial MDPI y la revista Computers and Electronics in Agriculture de la editorial Elsevier, las más activas en estos temas. 
También se mostró que la mayoría de los estudios primarios estudiaron la maleza, los cítricos y los arboles de palmera; las técnicas de aprendizaje más populares son las CNN y SVM. Con respecto al software utilizado para procesar las imágenes se ha utilizado desde lenguajes de programación de propósito general Matlab, Python y $\mathrm{C}++$, hasta software especializado como TerraScan, Agisoft PhotoScan y Pix4D. Con respecto al tipo de aeronave, mas del 74\% de los VANT utilizados en los estudios primarios fueron vehículos multirotor, destacando los de la marca DJI en particular el modelo Phantom 4. Con respecto a los sensores más utilizados la mayoría son del tipo RGB, multiespectral y LiDAR; con respecto a los estudios basados en cámaras multiespectrales se utilizaron principalmente los siguientes equipos: MicaSense, Canon S110 NIR, Tetracam y Parrot Sequoia.

Finalmente, se concluye que los VANT equipados con sensores basados en tecnología RGB, multiespectral y LiDAR son una excelente opción tecnológica para el desarrollo de aplicaciones agrícolas y forestales que ayuden a la obtención de mayores rendimientos económicos, sociales y medioambientales.

\section{Referencias}

Escalante Torrado, J. O., Aceres Jiménez, J. J., \& Porras Díaz, H. (2016). Ortomosaicos y modelos digitales de elevación generados a partir de imágenes tomadas con sistemas UAV. Tecnura, 20(50), 119-140.

De-la-Torre, M., Zatarain, O., Avila-George, H., Muñoz, M., Oblitas, J., Lozada, R., Mejía, J., \& Castro, W. (2019). Multivariate Analysis and Machine Learning for Ripeness Classification of Cape Gooseberry Fruits. Processes, 7(12), 928.

González, A., Amarillo, G., Amarillo, M., \& Sarmiento, F. (2016). Drones aplicados a la agricultura de precisión. Revista especializada en ingeniería, 10, 23-37.

Grenzdörffer, G. J., Engel, A., \& Teichert, B. (2008). The photogrammetric potential of low-cost UAVs in forestry and agriculture. The International Archives of the Photogrammetry Remote Sensing and Spatial Information Sciences, 31(B3), 1207-1214.

Hadas, E., Jozkow, G., Walicka, A., \& Borkowski, A. (2019). Apple orchard inventory with a LiDAR equipped unmanned aerial system. International Journal of Applied Earth Observation and Geoinformation, 82, 101911.

Hernández, L., Muñoz, M., Mejía, J., Peña, A., Rangel, N., \& Torres, C. (2017). Una revisión sistemática de la literatura enfocada en el uso de gamificación en equipos de trabajo en la ingeniería de software. RISTI-Revista Ibérica de Sistemas e Tecnologias de Informação, (21), 33-50.

Hunt, E. R., Hively, W. D., Fujikawa, S. J., Linden, D. S., Daughtry, C. S., \& McCarty, G. W. (2010). Acquisition of NIR-green-blue digital photographs from unmanned aircraft for crop monitoring. Remote Sensing, 2(1), 290-305.

Kitchenham, B., \& Charters, S. (2007). Guidelines for performing systematic literature reviews in software engineering. Keele University and Durham University, Technical report, ver. 2.3 EBSE. 
Koc-San, D., Selim, S., Aslan, N., \& San, B. T. (2018). Automatic citrus tree extraction from UAV images and digital surface models using circular Hough transform. Computers and electronics in agriculture, 150, 289-301.

Miramontes, J., Muñoz, M., Calvo-Manzano, J. A., \& Corona, B. (2016). Establecimiento del estado del arte sobre el aligeramiento de procesos de software. RISTI-Revista Ibérica de Sistemas e Tecnologias de Informação, (17), 16-25.

Muñoz, M., Peralta, M., \& Laporte, C. Y. (2019). Análisis de las debilidades que presentan las Entidades Muy Pequeñas al implementar el estándar ISO/IEC 29110: Una comparativa entre estado del arte y el estado de la práctica. RISTI-Revista Ibérica de Sistemas e Tecnologias de Informação, (34), 85-96.

She, T., Ehsani, R., Robbins, J., Leiva, J. N., \& Owen, J. (2014, July). Applications of small UAV systems for tree and nursery inventory management. In: Proceedings of the 12th International Conference on Precision Agriculture, Sacramento, CA, USA (pp. 20-23).

Tahar, K. N., \& Ahmad, A. (2013). An evaluation on fixed wing and multi-rotor UAV images using photogrammetric image processing. International Journal of Computer and Information Engineering, 7(1), 48-52.

Wohlin, C. (2014). Guidelines for snowballing in systematic literature studies and a replication in software engineering. In: Proceedings of the 18th international conference on evaluation and assessment in software engineering, London, England (pp. 1-10).

Wong, K. C. (2001). Survey of regional developments: Civil applications. In: UAV Australia Conference, Melbourne, Australia (pp. 8-9).

Anexo A: Estudios primarios

(EPo1) T. Adão, L. Pádua, J. Hruŝka, P. Marques, E. Peres, and J. J. Sousa, "A pilot digital image processing approach for detecting vineyard parcels in Douro region through high-resolution aerial imagery," Proceedings of the International Conference on Geoinformatics and Data Analysis - ICGDA '18. 2018.

(EPo2) Y. Bazi, S. Malek, N. Alajlan, and H. AlHichri, "An automatic approach for palm tree counting in UAV images," 2014 IEEE Geoscience and Remote Sensing Symposium. 2014.

(EPo3) S. Malek, Y. Bazi, N. Alajlan, H. AlHichri, and F. Melgani, "Efficient Framework for Palm Tree Detection in UAV Images," IEEE Journal of Selected Topics in Applied Earth Observations and Remote Sensing, vol. 7, no. 12. pp. 4692-4703, 2014.

(EPo4) L. Wallace, A. Lucieer, and C. S. Watson, "Evaluating Tree Detection and Segmentation Routines on Very High Resolution UAV LiDAR Data," IEEETransactions on Geoscience and Remote Sensing, vol. 52, no. 12. pp. 7619-7628, 2014.

(EPO5) J. R. Souza et al., "Automatic detection of Ceratocystis wilt in Eucalyptus crops from aerial images," 2015 IEEE International Conference on Robotics and Automation (ICRA). 2015. 
(EPo6) O. Guldogan et al., "Automated tree detection and density calculation using unmanned aerial vehicles," 2016 Visual Communications and Image Processing (VCIP). 2016.

(EPo7) G. L. A. Carrijo, D. E. Oliveira, G. A. de Assis, M. G. Carneiro, V. C. Guizilini, and J. R. Souza, “Automatic detection of fruits in coffee crops from aerial images," 2017 Latin American Robotics Symposium (LARS) and 2017 Brazilian Symposium on Robotics (SBR). 2017.

(EPo8) J. Ribera, Y. Chen, C. Boomsma, and E. J. Delp, “Counting plants using deep learning," 2017 IEEE Global Conference on Signal and Information Processing (GlobalSIP). 2017.

(EPo9) G. V. Nardari et al., "Crop Anomaly Identification with Color Filters and Convolutional Neural Networks," 2018 Latin American Robotic Symposium, 2018 Brazilian Symposium on Robotics (SBR) and 2018 Workshop on Robotics in Education (WRE). 2018.

(EP10) H. C. Oliveira, V. C. Guizilini, I. P. Nunes, and J. R. Souza, "Failure Detection in Row Crops From UAV Images Using Morphological Operators,” IEEE Geoscience and Remote Sensing Letters, vol. 15, no. 7. pp. 991-995, 2018.

(EP11) H. Huang, X. Li, and C. Chen, "Individual Tree Crown Detection and Delineation From Very-High-Resolution UAV Images Based on Bias Field and Marker-Controlled Watershed Segmentation Algorithms," IEEE Journal of Selected Topics in Applied Earth Observations and Remote Sensing, vol. 11, no. 7. pp. 2253-2262, 2018.

(EP12) Z. Fan, J. Lu, M. Gong, H. Xie, and E. D. Goodman, "Automatic Tobacco Plant Detection in UAV Images via Deep Neural Networks," IEEE Journal of Selected Topics in Applied Earth Observations and Remote Sensing, vol. 11, no. 3 . pp. 876-887, 2018.

(EP13) A. P et al., "CNN Based Technique for Automatic Tree Counting Using Very High Resolution Data,” 2018 International Conference on Design Innovations for $3 \mathrm{Cs}$ Compute Communicate Control (ICDI3C). 2018.

(EP14) A. O. Ok and A. Ozdarici-Ok, "Combining Orientation Symmetry and LM Cues for the Detection of Citrus Trees in Orchards From a Digital Surface Model," IEEE Geoscience and Remote Sensing Letters, vol. 15, no. 12. pp. 1817-1821, 2018.

(EP15) I. Ahmed et al., "Automatic Detection and Segmentation of Lentil Crop Breeding Plots From Multi-Spectral Images Captured by UAV-Mounted Camera," 2019 IEEE Winter Conference on Applications of Computer Vision (WACV). 2019.

(EP16) Bouachir, W., Ihou, K. E., Gueziri, H. E., Bouguila, N., \& Bélanger, N. (2019). "Computer Vision System for Automatic Counting of Planting Microsites Using UAV Imagery”. IEEE Access, 7, 82491-82500.

(EP17) Kitano, B. T., Mendes, C. C., Geus, A. R., Oliveira, H. C., \& Souza, J. R. (2019). "Corn Plant Counting Using Deep Learning and UAV Images". IEEE Geoscience and Remote Sensing Letters. 
(EP18) da Silva Vieira, G., Rocha, B. M., Soares, F., Lima, J. C., Pedrini, H., Costa, R., \& Ferreira, J. (2019, November). "Extending the Aerial Image Analysis from the Detection of Tree Crowns". In 2019 IEEE 31st International Conference on Tools with Artificial Intelligence (ICTAI) (pp. 1681-1685). IEEE.

(EP19) Yancho, J. M. M., Coops, N. C., Tompalski, P., Goodbody, T. R., \& Plowright, A. (2019). "Fine-Scale Spatial and Spectral Clustering of UAV-Acquired Digital Aerial Photogrammetric (DAP) Point Clouds for Individual Tree Crown Detection and Segmentation". IEEE Journal of Selected Topics in Applied Earth Observations and Remote Sensing, 12(10), 4131-4148.

(EP2O) Windrim, L., \& Bryson, M. (2018). "Forest Tree Detection and Segmentation using High Resolution Airborne LiDAR”. IEEE/RSJ International Conference on Intelligent Robots and Systems (pp. 3898-3905).

(EP21) Pignatti, S., Casa, R., Harfouche, A., Huang, W., Palombo, A., \& Pascucci, S. (2019, July). "Maize Crop and Weeds Species Detection by Using UAV VNIR Hyperpectral Data”. In IGARSS 2019-2019 IEEE International Geoscience and Remote Sensing Symposium (pp. 7235-7238). IEEE.

(EP22) Morales, V. A., Galvis, J. R., Garcia, E. G., \& Salcedo, I. L. (2019, July). "Estimation of Individual Potato Plants Area and Volume From UAV-Based Multispectral Images”. In IGARSS 2019-2019 IEEE International Geoscience and Remote Sensing Symposium (pp. 6259-6262). IEEE.

(EP23) L. Wallace, A. Lucieer, C. Watson, and D. Turner, "Development of a UAVLiDAR System with Application to Forest Inventory,” Remote Sensing, vol. 4, no. 6. pp. 1519-1543, 2012.

(EP24) J. M. Peña, J. Torres-Sánchez, A. Serrano-Pérez, A. I. de Castro, and F. LópezGranados, "Quantifying efficacy and limits of unmanned aerial vehicle (UAV) technology for weed seedling detection as affected by sensor resolution," Sensors , vol. 15, no. 3, pp. 5609-5626, Mar. 2015.

(EP25) S. Puliti, H. Ørka, T. Gobakken, and E. Næsset, "Inventory of Small Forest Areas Using an Unmanned Aerial System," Remote Sensing, vol. 7, no. 8. pp. 9632-9654, 2015.

(EP26) C. Poblete-Echeverría, G. Olmedo, B. Ingram, and M. Bardeen, "Detection and Segmentation of Vine Canopy in Ultra-High Spatial Resolution RGB Imagery Obtained from Unmanned Aerial Vehicle (UAV): A Case Study in a Commercial Vineyard,” Remote Sensing, vol. 9, no. 3. p. 268, 2017.

(EP27) H. Jiang, S. Chen, D. Li, C. Wang, and J. Yang, "Papaya Tree Detection with UAV Images Using a GPU-Accelerated Scale-Space Filtering Method,” Remote Sensing, vol. 9, no. 7. p. 721, 2017.

(EP28) O. Nevalainen et al., "Individual Tree Detection and Classification with UAV-Based Photogrammetric Point Clouds and Hyperspectral Imaging," Remote Sensing, vol. 9, no. 3. p. 185, 2017. 
(EP29) J. Albetis et al., "Detection of Flavescence dorée Grapevine Disease Using Unmanned Aerial Vehicle (UAV) Multispectral Imagery,” Remote Sensing, vol. 9, no. 4. p. 308, 2017.

(EP30) T. K. Alexandridis et al., "Novelty Detection Classifiers in Weed Mapping: Silybum marianum Detection on UAV Multispectral Images,” Sensors , vol. 17, no. 9, Sep. 2017.

(EP31) M. Mohan et al., "Individual Tree Detection from Unmanned Aerial Vehicle (UAV) Derived Canopy Height Model in an Open Canopy Mixed Conifer Forest," Forests, vol. 8, no. 9. p. 340, 2017.

(EP32) O. Csillik, J. Cherbini, R. Johnson, A. Lyons, and M. Kelly, "Identification of Citrus Trees from Unmanned Aerial Vehicle Imagery Using Convolutional Neural Networks," Drones, vol. 2, no. 4. p. 39, 2018.

(EP33) M. Hassanein, Z. Lari, and N. El-Sheimy, "A New Vegetation Segmentation Approach for Cropped Fields Based on Threshold Detection from Hue Histograms," Sensors, vol. 18, no. 4, Apr. 2018.

(EP34) L. Han et al., "Quantitative Identification of Maize Lodging-Causing Feature Factors Using Unmanned Aerial Vehicle Images and a Nomogram Computation," Remote Sensing, vol. 10, no. 10. p. 1528, 2018.

(EP35) J. Yeom, J. Jung, A. Chang, M. Maeda, and J. Landivar, "Automated Open Cotton Boll Detection for Yield Estimation Using Unmanned Aircraft Vehicle (UAV) Data,” Remote Sensing, vol. 10, no. 12. p. 1895, 2018.

(EP36) X. Wu, X. Shen, L. Cao, G. Wang, and F. Cao, “Assessment of Individual Tree Detection and Canopy Cover Estimation using Unmanned Aerial Vehicle based Light Detection and Ranging (UAV-LiDAR) Data in Planted Forests," Remote Sensing, vol. 11, no. 8. p. 908, 2019.

(EP37) J. Wu, G. Yang, X. Yang, B. Xu, L. Han, and Y. Zhu, "Automatic Counting of in situ Rice Seedlings from UAV Images Based on a Deep Fully Convolutional Neural Network," Remote Sensing, vol. 11, no. 6. p. 691, 2019.

(EP38) E. Salamí, A. Gallardo, G. Skorobogatov, and C. Barrado, "On-the-Fly Olive Trees Counting Using a UAS and Cloud Services,” Remote Sensing, vol. 11, no. 3 . p. 316, 2019.

(EP39) A. Safonova, S. Tabik, D. Alcaraz-Segura, A. Rubtsov, Y. Maglinets, and F. Herrera, "Detection of Fir Trees (Abies sibirica) Damaged by the Bark Beetle in Unmanned Aerial Vehicle Images with Deep Learning,” Remote Sensing, vol. 11, no. 6. p. 643, 2019.

(EP4O) Lin, Q., Huang, H., Wang, J., Huang, K., \& Liu, Y. (2019). “Detection of Pine Shoot Beetle (PSB) Stress on Pine Forests at Individual Tree Level using UAV-Based Hyperspectral Imagery and Lidar”. Remote Sensing, 11(21), 2540.

(EP41) Zhou, J., Tian, Y., Yuan, C., Yin, K., Yang, G., \& Wen, M. (2019). “Improved UAV Opium Poppy Detection Using an Updated YOLOv3 Model”. Sensors, 19(22), 4851. 
(EP42) Salamí, E., Gallardo, A., Skorobogatov, G., \& Barrado, C. (2019). "On-the-fly olive tree counting using a UAS and cloud services”. Remote Sensing, 11(3), 316.

(EP43) C. Hung, M. Bryson, and S. Sukkarieh, "Multi-class predictive template for tree crown detection,” ISPRS Journal of Photogrammetry and Remote Sensing, vol. 68. pp. 170-183, 2012.

(EP44) R. Calderón, J. A. Navas-Cortés, C. Lucena, and P. J. Zarco-Tejada, "Highresolution airborne hyperspectral and thermal imagery for early detection of Verticillium wilt of olive using fluorescence, temperature and narrow-band spectral indices," Remote Sensing of Environment, vol. 139. pp. 231-245, 2013.

(EP45) R. A. Diaz-Varela, P. J. Zarco-Tejada, V. Angileri, and P. Loudjani, “Automatic identification of agricultural terraces through object-oriented analysis of very high resolution DSMs and multispectral imagery obtained from an unmanned aerial vehicle,” J. Environ. Manage., vol. 134, pp. 117-126, Feb. 2014.

(EP46) P. J. Zarco-Tejada, R. Diaz-Varela, V. Angileri, and P. Loudjani, "Tree height quantification using very high resolution imagery acquired from an unmanned aerial vehicle (UAV) and automatic 3D photo-reconstruction methods," European Journal of Agronomy, vol. 55. pp. 89-99, 2014.

(EP47) Y. Lin, M. Jiang, Y. Yao, L. Zhang, and J. Lin, "Use of UAV oblique imaging for the detection of individual trees in residential environments," Urban Forestry \& Urban Greening, vol. 14, no. 2. pp. 404-412, 2015.

(EP48) M. Pérez-Ortiz, J. M. Peña, P. A. Gutiérrez, J. Torres-Sánchez, C. HervásMartínez, and F. López-Granados, "A semi-supervised system for weed mapping in sunflower crops using unmanned aerial vehicles and a crop row detection method," Applied Soft Computing, vol. 37. pp. 533-544, 2015.

(EP49) J. Torres-Sánchez, F. López-Granados, and J. M. Peña, “An automatic objectbased method for optimal thresholding in UAV images: Application for vegetation detection in herbaceous crops," Computers and Electronics in Agriculture, vol. 114. pp. 43-52, 2015 .

(EP50) L. Comba, P. Gay, J. Primicerio, and D. R. Aimonino, "Vineyard detection from unmanned aerial systems images," Computers and Electronics in Agriculture, vol. 114. pp. 78-87, 2015.

(EP51) O. Hassaan, A. K. Nasir, H. Roth, and M. Fakhir Khan, "Precision Forestry: Trees Counting in Urban Areas Using Visible Imagery based on an Unmanned Aerial Vehicle," IFAC-PapersOnLine, vol. 49, no. 16. pp. 16-21, 2016.

(EP52) A. dos S. Ferreira, A. dos Santos Ferreira, D. M. Freitas, G. G. da Silva, H. Pistori, and M. T. Folhes, "Weed detection in soybean crops using ConvNets," Computers and Electronics in Agriculture, vol. 143. pp. 314-324, 2017.

(EP53) S. Puliti, L. T. Ene, T. Gobakken, and E. Næsset, "Use of partial-coverage UAV data in sampling for large scale forest inventories," Remote Sensing of Environment, vol. 194. pp. 115-126, 2017. 
(EP54) J. Gao et al., "Fusion of pixel and object-based features for weed mapping using unmanned aerial vehicle imagery," International Journal of Applied Earth Observation and Geoinformation, vol. 67. pp. 43-53, 2018.

(EP55) V. Otero et al., "Managing mangrove forests from the sky: Forest inventory using field data and Unmanned Aerial Vehicle (UAV) imagery in the Matang Mangrove Forest Reserve, peninsular Malaysia,” Forest Ecology and Management, vol. 411. pp. 35-45, 2018.

(EP56) R. Näsi et al., "Remote sensing of bark beetle damage in urban forests at individual tree level using a novel hyperspectral camera from UAV and aircraft," Urban Forestry \& Urban Greening, vol. 30. pp. 72-83, 2018.

(EP57) L. Comba, A. Biglia, D. R. Aimonino, and P. Gay, "Unsupervised detection of vineyards by $3 \mathrm{D}$ point-cloud UAV photogrammetry for precision agriculture," Computers and Electronics in Agriculture, vol. 155. pp. 84-95, 2018.

(EP58) D. Koc-San, S. Selim, N. Aslan, and B. T. San, "Automatic citrus tree extraction from UAV images and digital surface models using circular Hough transform," Computers and Electronics in Agriculture, vol. 150. pp. 289-301, 2018.

(EP59) D. Yin and L. Wang, "Individual mangrove tree measurement using UAV-based LiDAR data: Possibilities and challenges," Remote Sensing of Environment, vol. 223. pp. 34-49, 2019.

(EP6o) K. S. Ochoa and Z. Guo, "A framework for the management of agricultural resources with automated aerial imagery detection," Computers and Electronics in Agriculture, vol. 162. pp. 53-69, 2019.

(EP61) Ochoa, K. S., \& Guo, Z. (2019). “A framework for the management of agricultural resources with automated aerial imagery detection”. Computers and Electronics in Agriculture, 162, 53-69.

(EP62) Andújar, D., Moreno, H., Bengochea-Guevara, J. M., de Castro, A., \& Ribeiro, A. (2019). "Aerial imagery or on-ground detection? An economic analysis for vineyard crops”. Computers and electronics in agriculture, 157, 351-358.

(EP63) Hadas, E., Jozkow, G., Walicka, A., \& Borkowski, A. (2019). “Apple orchard inventory with a LiDAR equipped unmanned aerial system”. International Journal of Applied Earth Observation and Geoinformation, 82, 101911.

(EP64) Ampatzidis, Y., Partel, V., Meyering, B., \& Albrecht, U. (2019). "Citrus rootstock evaluation utilizing UAV-based remote sensing and artificial intelligence”. Computers and Electronics in Agriculture, 164, 104900.

(EP65) Madec, S., Jin, X., Lu, H., De Solan, B., Liu, S., Duyme, F., \& Baret, F. (2019). "Ear density estimation from high resolution RGB imagery using deep learning technique”. Agricultural and forest meteorology, 264, 225-234.

(EP66) Torres, I., Sánchez, M. T., Cho, B. K., Garrido-Varo, A., \& Pérez-Marín, D. (2019). "Setting up a methodology to distinguish between green oranges and leaves using hyperspectral imaging”. Computers and Electronics in Agriculture, 167, 105070. 
(EP67) Stefas, N., Bayram, H., \& Isler, V. (2019). "Vision-based monitoring of orchards with UAVs". Computers and Electronics in Agriculture, 163, 104814.

(EP68) M. Pérez-Ortiz, P.A. Gutiérrez, J. M. Peña, J. Torres-Sánchez, C. Hervás-Martínez, and F. López-Granados, "An Experimental Comparison for the Identification of Weeds in Sunflower Crops via Unmanned Aerial Vehicles and Object-Based Analysis," Advances in Computational Intelligence. pp. 252-262, 2015.

(EP69) I. H. Yano, J. R. Alves, W. E. Santiago, and B. J. T. Mederos, "Identification of weeds in sugarcane fields through images taken by UAV and Random Forest classifier," IFAC-PapersOnLine, vol. 49, no. 16. pp. 415-420, 2016.

(EP70) R. A. Diaz-Varela, P. J. Zarco-Tejada, V. Angileri, and P. Loudjani, “Automatic identification of agricultural terraces through object-oriented analysis of very high resolution DSMs and multispectral imagery obtained from an unmanned aerial vehicle,” J. Environ. Manage., vol. 134, pp. 117-126, Feb. 2014.

(EP71) M. K. Hentz, A. P. D. Corte, S. P. Netto, M. P. Strager, and E. R. Schoeninger, "Treedetection: automatic tree detection using UAV-based data," Floresta, vol. 48, no. 3. p. 393, 2018.

(EP72) M. Bah, A. Hafiane, and R. Canals, "Deep Learning with Unsupervised Data Labeling for Weed Detection in Line Crops in UAV Images," Remote Sensing, vol. 10, no. 11. p. 1690, 2018.

(EP73) Y. Wang, X. Zhu, and B. Wu, "Automatic detection of individual oil palm trees from UAV images using HOG features and an SVM classifier," International Journal of Remote Sensing. pp. 1-15, 2018.

(EP74) R. Kestur, A. Angural, B. Bashir, S. N. Omkar, G. Anand, and M. B. Meenavathi, "Tree Crown Detection, Delineation and Counting in UAV Remote Sensed Images: A Neural Network Based Spectral-Spatial Method," Journal of the Indian Society of Remote Sensing, vol. 46, no. 6. pp. 991-1004, 2018.

(EP75) K. Kuželka and P. Surový, “Automatic detection and quantification of wild game crop damage using an unmanned aerial vehicle (UAV) equipped with an optical sensor payload: a case study in wheat," European Journal of Remote Sensing, vol. 51, no. 1. pp. 241-250, 2018.

(EP76) N. Demir, "Using UAVs for detection of trees from digital surface models," Journal of Forestry Research, vol. 29, no. 3. pp. 813-821, 2018.

(EP77) J. A. Thomasson et al., "Disease detection and mitigation in a cotton crop with UAV remote sensing," Autonomous Air and Ground Sensing Systems for Agricultural Optimization and Phenotyping III. 2018.

(EP78) M. Basso and E. P. de Freitas, "A UAV Guidance System Using Crop Row Detection and Line Follower Algorithms," Journal of Intelligent \& Robotic Systems. 2019.

(EP79) S. Selim, N. K. Sonmez, M. Coslu, and I. Onur, "Semi-automatic Tree Detection from Images of Unmanned Aerial Vehicle Using Object-Based Image Analysis Method," Journal of the Indian Society of Remote Sensing, vol. 47, no. 2. pp. 193-200, 2019. 
(EP80) Hassanein, M., Khedr, M., \& El-Sheimy, N. (2019). “Crop row detection procedure using low-cost uav imagery system”. International Archives of the Photogrammetry, Remote Sensing \& Spatial Information Sciences.

(EP81) Neupane, B., Horanont, T., \& Hung, N. D. (2019). “Deep learning based banana plant detection and counting using high-resolution red-green-blue (RGB) images collected from unmanned aerial vehicle (UAV)". PloS one, 14(10).

(EP82) Dash, J. P., Watt, M. S., Paul, T. S., Morgenroth, J., \& Pearse, G. D. (2019). "Early detection of invasive exotic trees using UAV and manned aircraft multispectral and LiDAR Data”. Remote Sensing, 11(15), 1812.

(EP83) Chakraborty, K., Saikom, V., Borah, S. B., Kalita, M., Gupta, C., Meitei, L. R., \& Raju, P. L. N. (2019). "Forest biometric parameter extraction using unmanned aerial vehicle to aid in forest inventory data collection". Current Science (00113891), 117(7).

(EP84) Harto, A. B., Prastiwi, P. A. D., Ariadji, F. N., Suwardhi, D., Dwivany, F. M., Nuarsa, I. W., \& Wikantika, K. (2019). "Identification of Banana Plants from Unmanned Aerial Vehicles (UAV) Photos Using Object Based Image Analysis (OBIA) Method (A Case Study in Sayang Village, Jatinangor District, West Java)". HAYATI Journal of Biosciences, 26(1), 7.

(EP85) Zaforemska, A., Xiao, W., \& Gaulton, R. (2019). "Individual tree detection from UAV Lidar data in a mixed species woodland”. ISPRS Geospatial Week 2019.

(EP86) Alekseev, A., Danilov, Y., Nikiforov, A., Guzuk, M., \& Kireev, D. (2019, August). "The use of unmanned aerial vehicle (UAV) for inventory and assessment of the experimental plantation in Lisino training and experimental forest of SaintPetersburg State Forest Technical University”. In IOP Conference Series: Earth and Environmental Science (Vol. 316, No. 1, p. 012004). IOP Publishing.

(EP87) Marques, P., Pádua, L., Adão, T., Hruška, J., Peres, E., Sousa, A., \& Sousa, J. J. (2019). "UAV-based automatic detection and monitoring of chestnut trees". Remote Sensing, 11(7), 855 .

(EP88) T. Kattenborn, M. Sperlich, K. Bataua, and B. Koch, "Automatic Single Tree Detection in Plantations using UAV-based Photogrammetric Point clouds," ISPRS - International Archives of the Photogrammetry, Remote Sensing and Spatial Information Sciences, vol. XL-3. pp. 139-144, 2014.

(EP89) M. Zortea, M. M. G. Macedo, A. B. Mattos, B. C. Ruga, and B. H. Gemignani, "Automatic Citrus Tree Detection from UAV Images based on Convolutional Neural Networks," in Conference on Graphics, Patterns and Images, Foz do Iguaçu.

(EP9o) M. B. Cruzan et al., "Small unmanned aerial vehicles (micro-UAVs, drones) in plant ecology," Appl. Plant Sci., vol. 4, no. 9, Sep. 2016. 